\title{
Genetic testing in dementia - utility and clinical strategies
}

Carolin A. M. Koriath ${ }^{1}$, Janna Kenny ${ }^{2}$, Nathalie S. Ryan ${ }^{3}$, Jonathan D. Rohrer ${ }^{3}$, Jonathan M. Schott ${ }^{3}$, Henry Houlden ${ }^{4}$, Nick C. Fox ${ }^{3,6}$, Sarah J. Tabrizi ${ }^{5}$ and Simon Mead ${ }^{1+}$

${ }^{1}$ MRC Prion Unit at UCL, UCL Institute of Prion Diseases, London, UK.

${ }^{2}$ South West Thames Regional Genetics Service, London, UK.

${ }^{3}$ Dementia Research Centre, Department of Neurodegenerative Disease, UCL Queen Square Institute of Neurology, London, UK.

${ }^{4}$ Neurogenetics Laboratory, National Hospital for Neurology and Neurosurgery, London, UK.

${ }^{5}$ Huntington's Disease Centre, Department of Neurodegenerative Disease, UCL Queen Square Institute of Neurology, London, UK.

${ }^{6}$ UK Dementia Research Institute, UCL Queen Square Institute of Neurology, London, UK.

†email: s.mead@prion.ucl.ac.uk

\begin{abstract}
Technology for clinical genetic testing in dementia disorders has advanced rapidly but remains to be more widely implemented in practice. A positive genetic test offers a precise molecular diagnosis, can help members of an affected family to determine personal risk, provides a basis for reproductive choices and offers options for clinical trials. The likelihood of identifying a genetic cause of dementia depends on the clinical condition, the age at onset and family history. Attempts to match phenotypes to single genes are mostly inadvisable owing to clinical overlap between the dementias, pleiotropy and concurrent mutations. Currently, the appropriate genetic test in most cases of dementia is next-generation sequencing gene panels, though some conditions necessitate specific types of test, such as repeat expansion testing. Whole-exome and whole-genome sequencing are becoming financially feasible but raise complex issues, such as variants of uncertain significance, secondary findings and the potential for re-analysis in light of new information. Capacity for data analysis and counselling is already restricting provision of genetic testing, and the need for both will increase. Patients and their relatives need to be given reliable information to enable them to make informed choices about tests, treatments and data sharing; the ability of patients with dementia to make decisions must be taken into account when providing this information.
\end{abstract}

\section{[H1] Introduction}

Neurodegenerative diseases account for most cases of dementia worldwide. Some of these diseases are common, such as Alzheimer disease (AD), and others are less common or rare, such as 
frontotemporal dementia (FTD) and prion diseases. All involve progressive accumulation of abnormal forms of brain proteins, a process that can begin over a decade before neuronal damage is detectable $e^{1-3}$. Biofluid and imaging biomarkers of these processes are available, but use of these tests in clinical practice is currently prompted by the development of symptoms, by which time molecular pathologies are often already widespread in the brain. Similarly, clinical trials have largely involved patients with dementia who already have advanced pathologies, which could be a hindrance to the discovery of disease-modifying treatments - evidence from animal and clinical studies suggests that disease-modifying treatments, when discovered, will work better if given earlier in the disease course ${ }^{4}$.

Sporadic neurodegenerative dementias generally occur in old age $^{5}$ and are highly polygenic ${ }^{6,7}$. However, a proportion of neurodegenerative dementias are Mendelian, providing an opportunity to make a precise diagnosis in the very early stages of disease and to identify presymptomatic carriers of causal mutations. These familial forms of dementia are being leveraged to study cutting-edge pharmacological therapies that have been developed on the basis of the pathophysiological mechanisms revealed by the genetic causes. Trials of approaches such as amyloid- $\beta$ antibody therapy in $A D^{8}, G R N$ replacement in $\mathrm{FTD}^{9}$, and gene-silencing in Huntington disease $(H D)^{10}$ offer hope to patients and will improve our understanding of disease mechanisms.

Genetic testing to identify individuals with causative mutations is essential for trials of therapies in Mendelian dementias and the benefits for therapeutic development are clear. However, the justification for genetic testing in a clinical context is less obvious given the absence of proven disease-modifying therapies. Advances in testing technology and changes in public awareness have created several areas of uncertainty around when to offer testing and how best to do so ${ }^{11,12}$. In this Review, we consider the currently available genetic technologies and their potential use in neurodegenerative dementias, and the strengths and weaknesses of each. We consider the clinical presentations of dementia and how their differences and pathological associations determine the most appropriate form of testing. We also explore the universal challenges of clinical genomics, including variants of uncertain significance and secondary findings, the duty to update findings based on new discoveries, the ethics of consent, and the rights of relatives. We focus on Mendelian forms of dementia, as the use of common risk variants, either alone or in polygenic risk scores, is not yet established in clinical practice. Specifically, we focus on Mendelian forms of AD and FTD as the typical forms of dementia but also discuss atypical parkinsonian syndromes (which partially overlap with FTD) and rarer causes of dementia, including prion disease and HD, which warrant a different approach to testing. We do not consider vascular dementia and dementia with Lewy bodies, for which few specific genetic factors are known ${ }^{13,14}$.

\section{[H1] Sequencing technologies and their use}

Genetic testing is currently underused in clinical practice and there is considerable geographical variability in its use, both internationally and within countries ${ }^{15,16}$. Nevertheless, increasing availability of genetic testing and growing knowledge about genes and pathways implicated in dementia are transforming diagnostic genetic testing from a niche technique to a mainstream clinical tool. 


\section{[H2] Gene panel testing}

The most important recent development in methodology for gene testing is the capability to sequence multiple genes simultaneously using so-called next-generation sequencing (NGS). The most common application of NGS in diagnostic genetic testing is the use of gene panels - targeted sequencing of a set of genes with similar associated clinical phenotypes, often including rarer causes of disease (Error! Reference source not found.Error! Reference source not found.). Most laboratories use focused gene panels, either by sequencing amplicon-selected regions of interest or by performing whole-exome sequencing (WES) ${ }^{21}$ or whole-genome sequencing (WGS) and subsequently restricting their analysis to genes that are selected on the basis of the referral diagnosis. A dementia gene panel includes most genes linked to the phenotype - that is, the AD genes APP, PSEN1 and PSEN2 and the canonical FTD genes GRN and MAPT - but also genes associated with rarer causes of familial dementia and leukencephalopathy ${ }^{17}$.

\section{[H2] Whole-exome and whole-genome sequencing}

NGS techniques include WES and WGS (Error! Reference source not found.Error! Reference source not found. $)^{18}$. The exome is the $1 \%$ of the genome that comprises all human exons - that is, the DNA that is transcribed to produce mature mRNA. The exome is thought to contain the vast majority of mutations that cause inherited human disease ${ }^{19}$. The costs of WES and WGS have been falling rapidly, making these techniques financially feasible clinical testing methods. WES and WGS are particularly useful for highly heterogeneous disorders ${ }^{20,21}$. Compared with use of targeted gene panels, use of WES and, particularly WGS, can reduce bias and increase diagnostic capabilities; for example, these approaches can detect structural variations such as duplications, deletions, insertions and inversions of DNA, and enable data re-analysis should new clinical information become available or a new gene-disease association be discovered. They also make novel gene discovery possible.

Despite their benefits, some problems exist with WES and WGS. Both are still substantially more expensive than gene panels, especially the analysis and storage of large amounts of data. They can also raise the issue of incidental findings in genes that are unrelated to the suspected disease ${ }^{22,23}$. These issues are exacerbated with WGS, which is more expensive than WES and generates $\sim 100$-fold more data. Nonetheless, WGS offers the advantage of interrogating not only the exome but also non-coding regions, such as splice sites, promoters and regulatory sequences, where an increasing number of pathogenic mutations are now being identified ${ }^{20,24-26}$. In addition, WGS provides more homogeneous coverage with more accurate genotyping, is less susceptible to allelic drop out, and identifies more variants ${ }^{27,28}$. Reported rates of diagnosis with use of WES are $>25 \%$ for neurological disease ${ }^{20,29-31}$, and this rate is likely to be higher with use of WGS $^{32}$.

\section{[H2] Overcoming limitations of NGS}

The existing NGS techniques excel at detecting single nucleotide polymorphisms (SNPs) and small insertions and deletions. However, as a result of sequence assembly by aligning overlapping fragments with a reference genome, detection of larger deletions and insertions, copy number variants and tandem repeat disorders is hampered by the achievable read length in standard WGS, as is recognition of structural variants if their breakpoints are located in a repeat region ${ }^{33,34}$ (Error! Reference source not found.). Tools for detection of copy number variants perform poorly with WES 
data, so other methods should be employed for detection of these variants ${ }^{35}$. Bioinformatics analysis methods have been developed to overcome these difficulties with some success, especially with data from modern PCR-free WGS or from paired-read sequencing ${ }^{36-38}$, but these methods are not yet reliable enough to be used on a larger scale or in a clinical setting ${ }^{37}$. Fragment analysis, repeat prime PCR, and Southern blotting (Table 1) are still needed to confirm the presence of expansions and their size.

Long-read sequencing platforms have been developed in an attempt to overcome the limitations of current NGS (Table 1$)^{39}$. The read lengths of typical NGS platforms peak at several hundred base pairs, whereas long-read sequencing platforms can reach several thousand base pairs and sequence, at least partially, through long nucleotide repeat expansions ${ }^{39}$. However, these platforms cannot yet match the accuracy and reliability of more established platforms for detection of SNPs ${ }^{40,41}$.

\section{[H2] Towards mainstream genetic medicine}

The availability of genetic testing in mainstream clinical practice is increasing but varies between countries. In the UK, links between the National Health Service (NHS) and research laboratories have enabled relatively easy access to genetic testing compared with other countries, where the need for insurance funding or limited availability of genetics services can make testing inaccessible to many. Supporting equity of access in the UK is the NHS Genomic Test Directory ${ }^{42}$, a publicly available list of available tests, indications and who can access the tests. The available tests were previously restricted to single-gene tests or gene panels because WES and WGS were unjustifiably expensive, but these tests are now also being implemented. Indeed, in neonatal and paediatric intensive care, rapid WGS has already been implemented in the UK. The turnaround time is $\sim 1$ week and the rate of molecular diagnosis is $42 \%{ }^{43}$ - this approach is transforming clinical care and illustrates what future care could look like in other specialties.

If parent-child trios are not available - as is usually the case with dementia, where the age of onset is late - the diagnostic rate of WES in adult simplex cases (across specialities) is typically $20-25 \%{ }^{44}$. A similar diagnostic rate $(22.1 \%)$ has been achieved with use of a targeted dementia panel that covered 17 genes and was supplemented with PCR-based tests of the C9orf72 expansion and PRNP octapeptide repeat alteration in a mixed referral-based cohort with dementia (AD, FTD and prion disease) $)^{24}$. However, the potential for later re-analysis with WES and WGS as new information about genes and variants of uncertain significance becomes available is not to be underestimated. For these reasons, many laboratories worldwide have moved to use of targeted WES-based panels, for which all genes are sequenced but only those associated with the conditions of interest are analysed. A similar approach with WGS could now be used for early-onset and familial dementia and other neurological disorders.

Analysis is limited in this way because the analysis of genome sequencing data is demanding - variants in known causative genes (for example, a dementia gene panel) need to be identified and interpreted, and copy number variations and repeat expansions need to be analysed. When a patient's phenotype is ambiguous and when genetic findings are unusual, multi-disciplinary clinical and diagnostic teams can be beneficial because additional clinical phenotypic details can confirm the diagnosis or indicate additional genes to examine; for example, leukodystrophy on MRI indicates analysis of a leukodystrophy panel, or a history of recessive inheritance in the family could 
substantiate an ambiguous homozygous mutation. Similarly, recruitment of additional unaffected and affected family members could increase the genetic yield by allowing segregation analysis of variants.

As dementia genetics advances, new technologies, such as long-read sequencing, will need to be incorporated into the diagnostic testing protocol to more effectively identify and determine the size of copy number variants and repeat expansions. Another important need is an international database of mutations in patients with dementia and controls of different ethnicities. This information is needed because parent-child trios are rarely available for testing. As a result, online population databases, such as GnomAD, have been critical for judging the pathogenicity of variants ${ }^{45,46}$. As the size and content of such databases increase, so will their utility and statistical power. Given that most probands whose genetic data constitutes these databases are tested in early and middle-aged adulthood, pathogenic variants in Mendelian dementia will, unlike those in rare paediatric diseases, inevitably end up in population databases because of the typically late age of onset and the possibility that penetrance of these variants is low. Disease prevalence and frequency of a given variant therefore needs to be taken into account when judging its likely pathogenicity ${ }^{46}$; consequently, greater statistical power of population databases contributes enormously to variant classification.

\section{[H1] Who to test and which test to use}

In the past 20 years, the number of genes known to cause dementia has increased considerably (revealing the genetic heterogeneity), as has our understanding that a spectrum of phenotypes can result from the same or different mutations in the same gene (pleiotropy) and of the clinical overlap between different dementia syndromes. For these reasons, different types of genetic testing are needed, depending on the clinical phenotype, whether a genetic, clinical or pathological diagnosis has been made in a relative, whether biomarkers of molecular pathology are available, the number

of implicated genes, and the type of mutation expected (AD, Alzheimer disease; FTD, frontotemporal dementia; PCR, polymerase chain reaction; SNP, single nucleotide polymorphism. 
Table 1).

Patients with dementia (or potentially antecedent syndromes or focal cognitive disorders) should be offered a full diagnostic assessment to determine their likely clinical diagnosis. This assessment should include a detailed neurological examination and multi-generation family history that can indicate the likelihood of an autosomal dominant trait with use of, for example, the modified Goldman score ${ }^{24,47,48}$ (Error! Reference source not found.Error! Reference source not found.) - in our experience, this score strongly predicts the likelihood of finding a genetic explanation for the disease ${ }^{24}$. An important pitfall to avoid is mistaking an incomplete family history or a history that includes unrelated early deaths, for a negative history, although even a negative family history does not preclude a genetic diagnosis. In a clinical diagnosis referral series of patients with a censored or negative family history but in which the prior expectation of a pathogenic variant was high, the discovery rate was $3.5 \%$ in $A D, 8.6 \%$ in FTD and $10.7 \%$ in prion disease ${ }^{24}$. The lack of family history in these patients could be due to factors such as the difficulty of accurate diagnosis, misdiagnosis, early deaths from other causes in previous generations and/or anticipation in some diseases. On this basis, a genetic test might be warranted even with a negative family history ${ }^{49}$; for example, this could be the case for patients with an early age at onset, a particular clinical syndrome that has a high rate of genetic causes, or a family history with likely diagnostic uncertainty owing to historical or geographical factors. Symptoms of other family members can also add to the diagnostic picture $^{50}$ and enable testing and diagnosis earlier in the course of the disease when symptoms can be still be equivocal but treatments are likely to be most effective.

Historically, predictive testing of unaffected relatives has not been recommended in the absence of a known familial mutation, particularly for conditions that have genetic and non-genetic or polygenic aetiologies. However, in conditions for which preventive action can be taken, such as breast cancer, testing of unaffected individuals is sometimes offered if the family history strongly suggests a monogenic aetiology. Such testing cannot rule out a genetic cause but a negative test reduces the likelihood, though the absolute risk reduction for an individual is hard to quantify. Similar strategies are likely to become more appropriate in dementia in future, particularly if progress is made with preventive therapeutics.

Testing for point mutations and small insertions or deletions that cause frameshifts or inframe protein changes can be performed on most genes and produce highly reliable results. However, testing one or even a handful of cherry-picked genes in a diagnostic setting is only suitable for disorders with distinctive clinical presentations, such as HD and prion disease, because the odds of detecting a causal variant are otherwise low. Furthermore, clinical overlap and gene pleiotropy mean that a clinical diagnosis does not reliably correspond to the expected genetic cause and vice versa (Error! Reference source not found.Error! Reference source not found.Error! Reference source not found.). In addition, even if single-gene testing identifies a causal mutation, it cannot detect concurrent mutations, which are found notable more often in FTD than expected by chance ${ }^{51-}$ 53. Furthermore, synergistic effects between novel variants and those that have not been unequivocally proven as deleterious variants can also lead to disease ${ }^{54}$. A computer algorithm has been developed to partially automate assessment of such double variants ${ }^{55}$, but tools like these have not yet been evaluated for their clinical utility and are not in widespread use. Unless for confirmatory testing, single-gene testing is therefore not recommended in AD or FTD. 
We recommend the introduction of dementia gene panel testing into routine neurological and geriatric care whenever a genetic cause could be plausible. Routine testing will increase the number of patients with a definite genetic diagnosis and will identify family members who are at risk, who would then be eligible for targeted treatment as and when it becomes available. A definitive genetic diagnosis can also reduce the number of other diagnostic investigations required and enable optimization of care and treatment. Changes in practice require new skills, can raise anxieties in patients and affected families and direct costs, and increase the need for counselling. A prospective evaluation of different strategies - perhaps a randomized trial of gene panel testing versus standard case - might be useful to establish the benefits or harms of a change in strategy.

\section{[H2] Alzheimer disease}

$A D$ is the most common cause of dementia, both early-onset (before age 65 years) ${ }^{56}$ and late-onset. $A D$ is often used as a default diagnosis for patients with dementia, so a family history of AD should be treated cautiously if it was not confirmed with a biomarker or by pathology. Genetic testing is useful for the diagnosis of autosomal dominant $A D$, but is not useful for sporadic $A D$, which has a mixed aetiology involving multiple risk variants. The prevalence of autosomal dominant $A D$ is not firmly established, but it probably accounts for $<1 \%$ of all cases ${ }^{57}$. Determining when and who to test for this condition requires an understanding of its clinical and genetic heterogeneity.

\section{[H3] Autosomal dominant Alzheimer disease}

Autosomal dominant $A D$ is typically an early-onset disease with onset in the fourth to sixth decades of life. The most common causes are mutations in PSEN1, PSEN2 and APP and duplication of APP; PSEN1 mutations are the most common. The mean age of onset is younger among people with PSEN1 mutations than those with APP mutations; PSEN2 mutations are relatively rare and are associated with a somewhat later age of onset ${ }^{58}$. Nevertheless, mutations in each of the causative genes can cause disease with an age of onset $>60$ years. Age of onset is relatively consistent within families and correlates with parental age of onset and the mean age of onset associated with the specific mutation ${ }^{58}$; nevertheless, age of onset can vary between families with the same mutation ${ }^{59}$. Reduced penetrance of mutations that cause autosomal dominant AD has been reported ${ }^{60-62}$, which indicates the existence of other, currently unknown, modifiers.

As with sporadic $A D$, most patients with autosomal dominant $A D$ present with progressive memory impairment. However, this typical amnestic syndrome can also be caused by non-AD genetic dementias, such as FTD due to MAPT mutations (with matching medial temporal lobe atrophy on $\mathrm{MRI}$, mimicking $A D^{63}$ ) or inherited prion disease caused by, for example, octapeptide repeat insertions in $P R N P^{64}$. Indeed, in a study in 2018, among patients with a clinical diagnosis of $A D$ who had or were likely to have a deleterious variant, genetic causes that are not associated with $A D$ were found in $>30 \%$; affected genes included MAPT, GRN, VCP, CSF1R, PRNP, SQSTM1, TARDBP and $C 9 o r f 72^{24}$. Conversely, atypical presentations of autosomal dominant $A D$ can occur, so that patients with mutations that cause $A D$ receive clinical diagnoses other than $A D$; for example, PSEN1 
mutations have been identified in patients who have been diagnosed with FTD, prion disease and dementia-motor syndromes ${ }^{24,65,66}$. Interestingly, the posterior cortical atrophy (PCA) variant of $A D$, which involves progressive impairment of higher visual processing, has a young age of onset but is almost invariably a sporadic condition ${ }^{67-69}$ and has only rarely been reported as possible autosomal dominant $A D$ (a novel PSEN1 variant) ${ }^{70}$.

Considerable clinical phenotype heterogeneity also exists in autosomal dominant $A D$, influenced by the affected gene, the specific variant and the affected functional domains. People with PSEN1 mutations can develop atypical cognitive deficits, including initial behavioural changes, language impairment, dyscalculia or a dysexecutive phenotype ${ }^{67}$, and PSEN2 mutations can lead to early delusions and hallucinations ${ }^{71}$. Motor symptoms, including spastic paraparesis, extrapyramidal and cerebellar signs, have been associated with mutations in PSEN1 but not in APP ${ }^{72}$. These atypical presentations, as well as later age at onset, more white matter hyperintensities on MRI and more severe cerebral amyloid angiopathy, are associated with PSEN1 mutations beyond codon $200^{67,73-76}$. APP duplications and mutations within the amyloid- $\beta$ coding domain can also lead to particularly severe CAA, recurrent cerebral haemorrhages, focal neurological symptoms and seizures ${ }^{77,78}$. Seizures can be an early feature of all autosomal dominant $A D$ - approximately one third of patients have a first seizure in the first 5 years of their illness, often preceded by myoclonus - but seizures tend to occur earliest in patients with APP duplications and later with PSEN1, PSEN2 and APP mutations ${ }^{67,79}$.

\section{[H3] Testing for autosomal dominant AD}

Given the wide phenotypic heterogeneity of autosomal dominant AD and the diversity of genes that can underlie the condition ${ }^{24}$, targeted gene panels, WES or WGS are the most appropriate choice for genetic testing in this context. Greater use of gene panels to investigate dementia in an unbiased manner is likely to make the breadth of the clinical phenotype of autosomal dominant AD more apparent.

We recommend offering genetic testing to patients with $A D$ who have a strong family history of dementia (modified Goldman score 1-2), regardless of the age of onset, and to patients with an age of onset $<60$ years. We consider genetic testing for patients of all ages who have a modified Goldman score of 3 and for those with an age of onset between 60 and 65 years, depending on individual patient factors, such as relatives with other neurodegenerative diseases or family histories that are limited owing to small family sizes, unrelated early deaths and geographical limits of communication.

This approach of positively weighting a relevant family history and early age at onset is based on data from 2018 , in which $17 \%$ of deleterious variants identified in patients with AD were in patients with an age of onset $\geq 65$ years. Of these patients, $42 \%$ had a relevant family history and the rest had an incomplete family history. By comparison, for patients with an age of onset between 60 and 65 years, $50 \%$ had a relevant family history ${ }^{24}$.

[H3] Risk loci and $A P O E$ 
In addition to the autosomal dominant mutations that make development of AD almost inevitable, over 30 genetic loci are known to modestly increase the risk of $A D^{80-83}$. Each of these risk loci alone increases the risk only slightly but, when combined with demographic factors, they can predict diagnosis of $A D$ with accuracies of over two-thirds ${ }^{84}$. The most well-known risk locus for sporadic $A D$, which also contributes most of the risk, is $A P O E$, which encodes apolipoprotein $E$ (ApoE). Three isoforms of ApoE exist: $\varepsilon 2, \varepsilon 3$ and $\varepsilon 4^{85,86}$. Possession of an $\varepsilon 2$ allele protects against $A D$ and $\varepsilon 4$ confers risk. The estimated risk conferred by an $\varepsilon 4$ allele varies between studies and ethnic groups, but reported odds ratios are 1.8-9.9 ${ }^{87-90}$ and, according to one study, people who are homozygous for the $\varepsilon 4$ allele have at least a 15 -fold higher risk of $A D$ than those who are homozygous for the $\varepsilon 3$ allele ${ }^{91}$.

Despite the associated increase in risk, the $\varepsilon 4$ allele is neither necessary nor sufficient to cause $A D$, so clinical genetic testing for $A P O E$ or other risk loci genotypes is not recommended. Nevertheless, some direct-to-consumer genetic testing companies offer APOE genotyping, usually with limited or no pre-test explanation of the implications of different results and no support or follow-up. This practice has caused considerable concern within the medical community, not least because individuals who have identified themselves as being at risk of $A D$ are increasingly being referred to clinics where such testing is not performed. Aside from recommending standard lifestyle modifications to reduce the overall risk of dementia and providing information about recruitment to research studies, no specific recommendations can be made to individuals who are positive for $A P O E$ $\varepsilon 4$. Guidelines for counselling and disclosure of $A P O E$ testing results in the context of clinical trials are currently being considered ${ }^{92}$.

\section{[H2] Frontotemporal dementia}

FTD is a heterogeneous disorder that encompasses multiple clinical and pathological conditions ${ }^{93}$. Approximately $30 \%$ of FTD is familial and most genetic causes are autosomal dominant ${ }^{94,95}$. Since Mendelian mutations in the MAPT gene were discovered to cause FTD in $1998^{96-98}$ (common variants in MAPT are associated with AD), mutations in multiple genes have been associated with FTD. Only two others are common causes of genetic FTD: mutations in GRN and hexanucleotide expansions in $C 9 o r f 72^{98}$. Mutations in other genes are rare causes of FTD; these genes include TBK1, VCP, CHMP2B, FUS, SQSTM1, TARDBP, CHCHD10, TIA1 and CCNF ${ }^{98}$.

Each causal gene is associated with different clinical phenotypes, including each of the canonical FTD syndromes (behavioural variant FTD (bvFTD) and the primary progressive aphasias (PPAs)), the FTD spectrum disorders (amyotrophic lateral sclerosis (ALS), corticobasal syndrome ${ }^{99}$ and progressive supranuclear palsy (PSP)) and non-FTD disorders including AD-like, Parkinson disease-like and HD-like phenotypes ${ }^{100}$. For example, ALS and FTD-ALS are associated with C9orf72 expansions rather than GRN or MAPT mutations, whereas PPA is more commonly associated with $G R N$ mutations; these are the strongest specific associations, and other associations are more subtle. Cognitive profiles differ according to whether GRN, MAPT or C9orf72 are affected. GRN mutations are associated with poor and rapidly worsening attention, MAPT mutations are associated with impaired memory function, and C9orf72 expansions are associated with global but relatively stable cognitive impairment ${ }^{99}$. Age at onset can be any time from adolescence ${ }^{100}$ and varies in all genetic forms; in FTD with GRN mutations or C9orf72 expansions, onset in family members ${ }^{100}$ can 
differ by $>20$ years. The heritability of FTD varies according to phenotype ${ }^{94}$. The most heritable form is bvFTD, followed by FTD-ALS, PPA (the nonfluent variant is more heritable than the semantic variant) and the atypical parkinsonian syndromes ${ }^{94,101-103}$.

We advocate offering testing to all people with bvFTD or FTD-ALS. Among people with PPA and the atypical parkinsonian syndromes, we recommend offering testing only to those with a strong family history (a modified Goldman score $<3$ ) because the chance of finding a genetic cause in these conditions without a family history is typically low ${ }^{104,105}$. We suggest that targeted panels and WES or WGS are combined with testing for the C9orf72 expansion ${ }^{24}$ because some families have pathogenic mutations in C9orf72 and one of the other FTD-related genes ${ }^{24,51}$. Large-scale cohort studies of people with or at-risk of genetic FTD ${ }^{106,107}$ have paved the way for clinical trials of GRN replacement therapy in genetic FTD that are now starting ${ }^{9,108-110}$ and are likely to increase uptake of genetic testing as many trials will require knowledge of genetic status.

\section{[H2] HD and prion disease}

Unlike in other forms of dementia, testing for mutations in a single gene is warranted in HD and prion disease, as the typical symptoms and presentations and, in many cases, the family history suggest the diagnosis even if a genetic diagnosis has not previously been made in the family.

Prion diseases - Creutzfeldt-Jakob-Disease (CJD), Gerstman-Scheinker-Straussler disease (GSS), fatal familial insomnia (FFI), octapeptide repeat insertions (OPRI)-related disease, and prion protein systemic amyloidosis - are defined by accumulation of abnormal prion protein ${ }^{46}$ and can usually be detected with Sanger sequencing. Approximately $10-15 \%$ of cases of prion disease are caused by a mutation in $P R N P^{64}$. CJD is the most common form and is defined by rapidly progressive dementia, myoclonus and ataxia. GSS involves a frontal syndrome and cerebellar ataxia with peripheral loss of sensation, and FFI causes fragmented sleep, gait abnormalities and autonomic symptoms. OPRIrelated disease is also known as HD-like 1 because patients can present with chorea or other movement disorders in addition to rapidly progressive dementia and psychiatric symptoms ${ }^{111}$. Prion protein systemic amyloidosis causes a late-onset hereditary sensory and autonomic neuropathy without dementia, comparable to familial amyloid polyneuropathy ${ }^{112}$. Features such as myoclonus or insomnia as well as rapid cognitive and physical deterioration typically lead to clinical suspicion of prion disease, complemented by specific investigations that can then prompt a focused genetic test.

$\mathrm{HD}$ is one of the most common autosomal dominant neurodegenerative disorders ${ }^{113,114}$. The disease is typically defined by a triad of progressive movement, cognitive and psychiatric symptoms ${ }^{115}$. For many clinicians, chorea is the defining feature of HD and unequivocal motor extrapyramidal symptoms are important for diagnosis, but symptoms can range from hyperkinetic to hypokinetic and patients often experience a prodromal phase that involves cognitive impairment, anxiety and depression ${ }^{116}$. HD was the first neurodegenerative disease for which a likely genetic location was identified via genetic linkage (and later confirmed) ${ }^{117}$ and has since become a model disease for the development of ethical guidelines for predictive testing ${ }^{118}$. HD is caused by a CAG triplet repeat expansion in the HTT gene on chromosome 4; the expansion can be detected with fragment analysis or Southern blot (Table 1$)^{119}$. Penetrance is incomplete if the CAG repeat number is 36-39 but complete if it is $>40^{120}$. The number of repeats inversely correlates with the age at onset and often increases from one generation to the next (known as anticipation), especially when paternally inherited, although other genetic modifiers, including DNA repair genes ${ }^{121}$ and gene 
promoters ${ }^{122}$, also influence age at onset. As a result of the distinctive combination of symptoms, the diagnostic rate for the HD test is high - when the test first became available, only $\sim 1 \%$ of patients with HD symptoms tested negative for the HTT expansion ${ }^{123}$. However, the negative test rate has increased because the low cost and ready availability of the test means clinicians often request it to exclude the disorder even if the clinical syndrome is atypical. If the HD test is negative, the differential diagnosis is wide ${ }^{115}$ and gene panel testing is often unsuccessful ${ }^{124}$.

If a genetic cause continues to be suspected after a negative test for either prion disease or HD, patients can be offered WES or WGS to improve diagnostic rates. These techniques should be supplemented by testing for the C9orf72 expansion, which is the most common cause of HD phenocopy syndromes ${ }^{125}$ (Error! Reference source not found.).

\section{[H1] Challenges and ethics}

\section{[H2] Variant classification}

Next-generation sequencing technology leads to identification of a large number of variants, which can be classified with the American College of Medical Genetics and Association of Molecular Pathology (ACMG-AMP) ${ }^{126}$ guidelines. In these guidelines, all available evidence - including population, variant and disease-specific databases - is used to classify variants into one of five categories: benign, likely benign, variant of uncertain significance, likely pathogenic or pathogenic (Box 3Error! Reference source not found.). For variants that are classified as likely pathogenic or pathogenic, diagnostic, predictive or prenatal testing can be offered to other family members. However, many variants are classified as variants of unknown significance because the evidence is either insufficient or conflicting. The classification criteria are conservative and designed to ensure that a variant remains a variant of uncertain significance unless compelling evidence shows that it is pathogenic or benign because the risks of misclassifying a variant are considerable. Nevertheless, a variant that is initially classified as a variant of uncertain significance could become re-classifiable as the evidence changes. No consensus exists about when, how often or by whom variants should be reconsidered and re-classified, but as population databases expand and use of WES and WGS increase, classification of variants will improve.

Evidence suggests that people in whom a variant of uncertain significance is identified find this information difficult to deal with psychologically, and no consensus exists about when patients should be informed about a variant of uncertain significance ${ }^{127,128}$. Local protocols for pre-testing and post-testing counselling on this issue are particularly important when patients are offered genetic testing for a condition.

One possible strategy for dealing with variants of uncertain significance would be to tell patients about some variants of uncertain significance with insufficient evidence for pathogenicity, primarily to enable testing for segregation in relatives or further functional studies, and to enable the treating physician to pursue updates about classification at follow-up assessments. This strategy could be particularly useful in the dementias, as their typical late onset usually precludes testing of patient-parent trios that are typical in paediatric genetic testing. 
In this context, databases of variants of uncertain significance could be helpful. GeneMatcher, an existing database of variants of uncertain significance, is designed to connect researchers and patients who are interested in variants in the same gene, but does not highlight variants with additional indicators of pathogenicity, such as rarity in the general population or in silico predictions of pathogenicity, and is not searchable for others' submissions unless they match to one's own submission ${ }^{129}$, which limits its accessibility and the identification of new mutational hotspots. A repository of such suspicious variants of uncertain significance that includes some general clinical information, such as the associated clinical condition and age at onset, would be a rich resource for scientists, as it could help to identify more associated genes and increase knowledge about gain-of-function and loss-of-function mechanisms, thereby directing drug discovery. The online resources that are currently available cannot be searched specifically for this type of variant of uncertain significance for which some information indicates pathogenicity ${ }^{130,131}$. Such a category of 'potentially pathogenic variants' might stimulate attempts to develop biochemical, cell biology or computational methods to resolve the roles of variants of uncertain significance for specific genes, which would be a major advance ${ }^{132}$.

Another problem with the current method of variant classification is that some variants were erroneously classified as pathogenic before the development of large population databases. Therefore, when curating clinical databases, caution must be exercised when looking at variants that were classified before population databases were established in $2016^{45}$. For example, some PRNP variants that were classified as pathogenic are too frequent in the population to be considered fully penetrant in such a rare disease disease ${ }^{46}$. Similar calculations have been done for early-onset $A D$ and FTD and some variants that are thought to be deleterious seem not to be fully penetrant ${ }^{24}$. For these reasons, public databases of variants are inadequate as the sole source of variant annotation; additional review of clinical data and manual collation of information is still necessary ${ }^{133}$.

In the context of variant classification, direct-to-consumer genetic tests can be a problem because interpretation of a mutation's pathogenicity requires consideration of personal and family history. As more people use direct-to-consumer genetic tests without being offered appropriate pretest or post-test counselling or adequate information about variants that are identified, the counselling burden on clinical services is likely to increase further.

\section{[H2] Secondary findlings}

As use of comprehensive genetic testing approaches, such as WES and WGS, increases, so too does the possibility of clinically relevant secondary, or incidental, findings. Secondary findings are mutations that are unrelated to the condition being tested for but could have implications for future health. Examples include, but are not limited to, mutations that predispose to cancer, aortopathy or arrhythmia, and mutations that cause autosomal recessive conditions. Study findings indicate that such mutations are identified in $4.6-12 \%$ of tested individuals ${ }^{134,135}$.

The ACMG currently recommends that if WES or WGS are used clinically, secondary findings in 59 specific genes should be looked for and reported ${ }^{136}$. The genes included are associated with diseases in which screening and/or early treatment can improve patient outcomes, so genes associated with dementia are not currently included. However, such genes could be included in 
future if treatments are developed, particularly if initiation of these treatments in early stages of the disease provides most benefit. Studies have shown that following the current ACMG recommendations would mean that secondary findings would need reporting to $~ 3 \%$ of people tested; the time and cost implications of returning secondary findings are therefore substantial ${ }^{137-139}$.

If patients are offered WES or WGS, specific consent needs to be obtained for the reporting of secondary findings and they must be given an opportunity to opt out. Studies have shown that patients are broadly in favour of the disclosure of secondary findings ${ }^{140-142}$. However, it is difficult to be sure that consent is fully informed when such a large number of genes are involved because the implications for each are different. In addition, the list of secondary findings that should be reported for children differs from that for adults, so consideration needs to be given to whether further secondary findings should be reported when these children reach adulthood and how this would be done.

\section{[H2] Consent and counselling}

When genetic testing is considered, informed consent requires careful counselling of the patient, even if the test is diagnostic rather than predictive. The term counselling does not imply that this process only involves genetic counsellors - practicing neurologists and geriatricians often perform this duty for their own diagnostic testing. Obtaining consent can be particularly challenging when the patient has impaired cognition. The decision to test an individual who lacks capacity to provide informed consent should be based on their best interests and should ideally involve discussion with family members to ascertain what the wishes of the patient in relation to genetic testing were before their disease progressed. Other factors to consider include whether the patient has the cognitive ability to understand the results and whether the results could exacerbate their condition; for example, if anxiety or psychiatric features are part of their presentation.

Diagnostic genetic testing of symptomatic patients is usually requested by treating physicians, but predictive testing for asymptomatic individuals with a family history has traditionally been the remit of clinical geneticists and genetic counsellors. The protocol for predictive testing in $\mathrm{HD}$ and genetic dementias provides a framework for such predictive testing - this protocol ensures that the individual is informed of the risks and benefits of testing, the implications for employment and insurance, the availability of screening and/or risk modifying treatment and the implications for offspring and other relatives ${ }^{48,118}$. A key feature of this protocol is multiple appointments, which provide individuals with time to consider the consequences of testing and the opportunity to change their decision about whether to undergo testing. A shortage of clinical geneticists and counsellors worldwide makes it difficult to provide these opportunities to everyone, but predictive testing is not without risk and should not be undertaken lightly ${ }^{143}$. If access to genetic counselling is impossible, atrisk individuals should be counselled by the testing clinician about the risks and benefits and should be given the opportunity to consider these aspects before proceeding. Consideration should also be given to the manner in which results are communicated and the arrangements for follow-up with patients whose test is positive. Particular caution, for example, additional counselling that might involve relatives or additional support, are needed in complex family situations, such as the testing of monozygotic twins or when testing an individual will provide a de facto result for a parent who has refused testing, and if patients are at risk of a poor psychological outcome after testing. 
A genetic diagnosis can offer many benefits but also carries risks (Box 4Error! Reference source not found.).Error! Reference source not found. Consequently, the decision for or against a predictive test can be distressing, and uptake is generally low; for example, uptake of predictive testing for HD is $5-20 \%$ among at-risk relatives of patients, and uptake among those at risk of prion disease is $\sim 25 \%{ }^{144}$. However, with the advent of treatment trials in genetic neurological diseases, such as HD, spinal muscular atrophy and transthyretin amyloidosis, and in dementia (for example, trials of GRN gene replacement) we (the UCLH Neurogenetics Laboratory) have seen an upturn of $\sim 50 \%$ in uptake of predictive testing. Even in conditions with available treatments, the counselling and testing process should still ideally follow the protocol for $\mathrm{HD}^{47,118}$ in a specialist neurogenetics clinic with close laboratory support - counselling should take place over three appointments and turnaround of the genetic test once blood has been taken should be rapid (ideally within 2 weeks). With an increase in uptake, however, a shortage of clinical geneticists and genetic counsellors worldwide could limit the availability of pre-test and post-test counselling. Another factor that has increased uptake of predictive testing is availability of pre-implantation genetic diagnosis (PGD) for a growing number of genetic conditions. This procedure involves genetic testing of blastomeres before reimplantation during IVF, and PGD can be carried out without genetic testing of the at-risk parent, a process known as exclusion PGD.

[H2] Implications for relatives

Genetic testing is distinct from other medical investigations because it frequently has implications for relatives of the person being tested. The principle of confidentiality in medicine holds in genetic testing but given that most people want to have genetic testing at least in part to assist other family members, confidentiality is rarely an issue. Occasionally, however, a patient declines to tell their relatives of a genetic diagnosis, which poses an enormous ethical problem. This scenario occurred in the legal case of ABC versus St George's Hospital NHS Trust in the UK, in which a patient with cognitive capacity refused to tell his children the result of his positive diagnostic test for HD. The judgement made clear that clinicians have a duty to balance the right of the patient to confidentiality with the right of an interested third party to be informed of results that affect them ${ }^{145,146}$; therefore, under certain circumstances and potentially after taking advice from an ethics committee, a clinician might be allowed to disclose such results without the patient's consent.

Estimates suggest that up to $20 \%$ of relatives are not informed of genetic test results that are relevant to them, and the reasons range from wishing to protect relatives to being unaware that the information was relevant ${ }^{60,147}$. This ethical area is clearly complex and if a patient is being asked to pass on potentially life-changing information to relative, the ability of the patient to make decisions must be considered. In the UK, the Joint Committee in Genomic Medicine has provided guidance on how to proceed when issues about data sharing arise ${ }^{148}$.

If the results of a genetic test are unlikely to change the management of a patient - for example, if treatment is unavailable and investigation and monitoring will not be changed - then the timing of genetic testing should also be carefully considered. For example, a genetic test is needed to clarify the risks to a patient's relatives but in some cases, for example if issues exist in relation to an individual's capacity to consent or if the results could be distressing to the patient, storage of DNA for later testing or collection of samples during autopsy might be more appropriate. 
However, such a delay can cause problems; for example, relatives might want to understand their risk before having children, or if the next of kin does not allow testing of samples after the patient's death, other relatives could be denied the chance to clarify their risk. Testing of unaffected individuals in the absence of a known familial mutation is rarely offered owing to the heterogeneous nature of many genetic conditions, uncertainty with respect to penetrance and limitations in the interpretation of many variants.

Finally, the results of predictive genetic tests can have implications for insurance. In the UK, members of the Association of British Insurers must abide by a code that prevents them from asking people to have predictive genetic tests or to disclose the results of predictive genetic tests. One exception is HD, for which disclosure of known genetic test results is mandatory for critical illness insurance amounts above specific thresholds ${ }^{149}$. In addition, insurers will not (in line with the code of conduct they abide by) ask for or take into account the results of predictive tests obtained in the course of scientific research. This code came into effect in October 2018 and will be reviewed every 3 years. Implications for insurance differ between countries, and relevant aspects should be discussed with patients as part of pre-test counselling for any predictive test.

\section{[H1] Conclusions}

Widespread genetic testing is transforming clinical practice. Though only select patient cohorts are currently eligible for the first trials of disease-modifying drugs in neurodegenerative disease, the number of patients who are eligible will only grow with the advent of effective preventive and therapeutic approaches. For most dementias, the most appropriate genetic test is a dementia gene panel, which can be performed by analysis of WES or WGS to enable subsequent analysis of further genetic information if appropriate, supplemented by testing for the C9orf72 expansion. For specific disorders with known single-gene causes, such as HD and prion diseases, single-gene tests remain a suitable choice. In future, long-read sequencing will enable simultaneous testing for SNPs and expansion disorders, though this technique is not yet sufficiently accurate or affordable for clinical practice. However, as greater numbers of patients are tested for deleterious variants in an increasing number of genes, secondary findings and variants of uncertain significance are bound to be identified more frequently, creating new challenges, such as an increased need for pre-test and post-test counselling and the need for re-analysis as new information becomes available. Ethical aspects, such as the ability of patients with dementia to provide consent and the rights of relatives, will need to evolve as personalized medicine based on genetic testing becomes the reality.

1. Tabrizi, S.J. et al. Biological and clinical changes in premanifest and early stage Huntington's disease in the TRACK-HD study: the 12-month longitudinal analysis. Lancet Neurol 10, 31-42 (2011).

2. Sperling, R.A. et al. Toward defining the preclinical stages of Alzheimer's disease: recommendations from the National Institute on Aging-Alzheimer's Association workgroups on diagnostic guidelines for Alzheimer's disease. Alzheimers Dement 7, 280-92 (2011).

3. Andrews, K.A. et al. Acceleration of hippocampal atrophy rates in asymptomatic amyloidosis. Neurobiol Aging 39, 99-107 (2016). 
4. Baazaoui, N. \& Iqbal, K. A Novel Therapeutic Approach to Treat Alzheimer's Disease by Neurotrophic Support During the Period of Synaptic Compensation. J Alzheimers Dis 62, 1211-1218 (2018).

5. Hou, Y. et al. Ageing as a risk factor for neurodegenerative disease. Nat Rev Neurol 15, 565581 (2019).

6. Jansen, I.E. et al. Genome-wide meta-analysis identifies new loci and functional pathways influencing Alzheimer's disease risk. Nat Genet 51, 404-413 (2019).

7. Gatz, M. et al. Role of genes and environments for explaining Alzheimer disease. Arch Gen Psychiatry 63, 168-74 (2006).

8. Bateman, R.J. et al. The DIAN-TU Next Generation Alzheimer's prevention trial: Adaptive design and disease progression model. Alzheimers Dement 13, 8-19 (2017).

9. Alector. Alector Initiates Phase 2 Trial of ALO01 in Patients with Frontotemporal Dementia. (GlobeNewsWire. https://www.globenewswire.com/newsrelease/2019/09/09/1912694/0/en/Alector-Initiates-Phase-2-Trial-of-AL001-in-Patientswith-Frontotemporal-Dementia.html, 2019).

10. Tabrizi, S.J., Ghosh, R. \& Leavitt, B.R. Huntingtin Lowering Strategies for Disease Modification in Huntington's Disease. Neuron 101, 801-819 (2019).

11. Atkins, E.R. \& Panegyres, P.K. The clinical utility of gene testing for Alzheimer's disease. Neurol Int 3, e1 (2011).

12. Falk, N., Cole, A. \& Meredith, T.J. Evaluation of Suspected Dementia. Am Fam Physician 97, 398-405 (2018).

13. Dichgans, M. \& Leys, D. Vascular Cognitive Impairment. Circ Res 120, 573-591 (2017).

14. Keogh, M.J. et al. Exome sequencing in dementia with Lewy bodies. Transl Psychiatry 6, e728 (2016).

15. Ngo, J. \& Holroyd-Leduc, J.M. Systematic review of recent dementia practice guidelines. Age Ageing 44, 25-33 (2015).

16. Stevens, J.C. et al. Familial Alzheimer's disease and inherited prion disease in the UK are poorly ascertained. J Neurol Neurosurg Psychiatry 82, 1054-7 (2011).

17. Beck, J. et al. Validation of next-generation sequencing technologies in genetic diagnosis of dementia. Neurobiol Aging 35, 261-5 (2014).

18. Nho, K. et al. Whole-exome sequencing and imaging genetics identify functional variants for rate of change in hippocampal volume in mild cognitive impairment. Mol Psychiatry 18, 7817 (2013).

19. Cooper DN, K.M., Antonorakis SE. The nature and mechanisms of human gene mutation. in The Metabolic and Molecular Bases of Inherited Disease (ed. Scriver C, B.a., Sly WS, Valle D) 259-291 (McGraw-Hill, New York, 1995).

20. Blauwendraat, C. et al. The wide genetic landscape of clinical frontotemporal dementia: systematic combined sequencing of 121 consecutive subjects. Genet Med 20, 240-249 (2018).

21. Fogel, B.L. Genetic and genomic testing for neurologic disease in clinical practice. Handb Clin Neurol 147, 11-22 (2018).

22. Ku, C.S. et al. Exome sequencing: Dual role as a discovery and diagnostic tool. Annals of Neurology 71, 5-14 (2012).

23. Rabbani, B., Tekin, M. \& Mahdieh, N. The promise of whole-exome sequencing in medical genetics. J Hum Genet 59, 5-15 (2014).

24. Koriath, C. et al. Predictors for a dementia gene mutation based on gene-panel nextgeneration sequencing of a large dementia referral series. Mol Psychiatry, Oct 2;10.1038/s41380-018-0224-0. doi: 10.1038/s41380-018-0224-0. Online ahead of print (2018).

This paper provides evidence-based recommendations on which patients with dementia to test with which test, and calculations on how frequent a variant can be in the population for 
the likelihood to be high that it is a fully penetrant variant that causes early-onset dementia.

25. DeJesus-Hernandez, M. et al. Expanded GGGGCC hexanucleotide repeat in noncoding region of C9ORF72 causes chromosome 9p-linked FTD and ALS. Neuron 72, 245-56 (2011).

26. Sposito, T. et al. Developmental regulation of tau splicing is disrupted in stem cell-derived neurons from frontotemporal dementia patients with the $10+16$ splice-site mutation in MAPT. Hum Mol Genet 24, 5260-9 (2015).

27. Björn Na, P.S., Sigurgeirsson B, Lundeberg J , Gréen H, Sahlén P. Comparison of Variant Calls from Whole Genome and Whole Exome Sequencing Data Using Matched Samples. Journal of Next Generation Sequencing \& Applications 5(2018).

28. Belkadi, A. et al. Whole-genome sequencing is more powerful than whole-exome sequencing for detecting exome variants. Proc Natl Acad Sci U S A 112, 5473-8 (2015).

29. Fogel, B.L. et al. Exome sequencing in the clinical diagnosis of sporadic or familial cerebellar ataxia. JAMA Neurol 71, 1237-46 (2014).

30. $\mathrm{Xu}, \mathrm{Y}$. et al. The Whole Exome Sequencing Clarifies the Genotype- Phenotype Correlations in Patients with Early-Onset Dementia. Aging Dis 9, 696-705 (2018).

31. Galatolo, D., Tessa, A., Filla, A. \& Santorelli, F.M. Clinical application of next generation sequencing in hereditary spinocerebellar ataxia: increasing the diagnostic yield and broadening the ataxia-spasticity spectrum. A retrospective analysis. Neurogenetics 19, 1-8 (2018).

32. Cuccaro, D., De Marco, E.V., Cittadella, R. \& Cavallaro, S. Copy Number Variants in Alzheimer's Disease. J Alzheimers Dis 55, 37-52 (2017).

33. Ebbert, M.T.W. et al. Long-read sequencing across the C9orf72 'GGGGCC' repeat expansion: implications for clinical use and genetic discovery efforts in human disease. $\mathrm{Mol}$ Neurodegener 13, 46 (2018).

34. Kumar, K.R., Cowley, M.J. \& Davis, R.L. Next-Generation Sequencing and Emerging Technologies. Semin Thromb Hemost 45, 661-673 (2019).

35. Zare, F., Dow, M., Monteleone, N., Hosny, A. \& Nabavi, S. An evaluation of copy number variation detection tools for cancer using whole exome sequencing data. BMC Bioinformatics 18, 286 (2017).

36. Dolzhenko, E. et al. Detection of long repeat expansions from PCR-free whole-genome sequence data. Genome Res 27, 1895-1903 (2017).

This study demonstrated how PCR-free whole-genome sequencing data can be analysed for trinucleotide repeat expansions.

37. Mousavi, N., Shleizer-Burko, S., Yanicky, R. \& Gymrek, M. Profiling the genome-wide landscape of tandem repeat expansions. Nucleic Acids Res 47, e90 (2019).

38. Wrzeszczynski, K.O. et al. Whole Genome Sequencing-Based Discovery of Structural Variants in Glioblastoma. Methods Mol Biol 1741, 1-29 (2018).

39. Mantere, T., Kersten, S. \& Hoischen, A. Long-Read Sequencing Emerging in Medical Genetics. Front Genet 10, 426 (2019).

40. Carneiro, M.O. et al. Pacific biosciences sequencing technology for genotyping and variation discovery in human data. BMC Genomics 13, 375 (2012).

41. Laver, T. et al. Assessing the performance of the Oxford Nanopore Technologies MinION. Biomol Detect Quantif 3, 1-8 (2015).

42. England, N. National Genomic Test Directory.

43. Mestek-Boukhibar, L. et al. Rapid Paediatric Sequencing (RaPS): comprehensive real-life workflow for rapid diagnosis of critically ill children. J Med Genet 55, 721-728 (2018).

44. Retterer, K. et al. Clinical application of whole-exome sequencing across clinical indications. Genet Med 18, 696-704 (2016).

45. Lek, M. et al. Analysis of protein-coding genetic variation in 60,706 humans. Nature 536, 285-91 (2016). 
46. Minikel, E.V. et al. Quantifying prion disease penetrance using large population control cohorts. Sci Transl Med 8, 322ra9 (2016).

In this study, elegant maths was used to demonstrate the importance of harnessing genetic variant population databases to assess variant pathogenicity because in a rare diseases, such as prion disease, some variants that are reported as pathogenic are too frequent in the population to be fully penetrant causal mutations.

47. Goldman, J.S. et al. An algorithm for genetic testing of frontotemporal lobar degeneration. Neurology 76, 475-83 (2011).

48. Goldman, J.S. Genetic testing and counseling in the diagnosis and management of youngonset dementias. Psychiatr Clin North Am 38, 295-308 (2015).

49. Deleon, J. \& Miller, B.L. Frontotemporal dementia. Handb Clin Neurol 148, 409-430 (2018).

50. Bird, T.D. \& Smith, C.O. Clinical approach to the patient with neurogenetic disease. Handb Clin Neurol 147, 3-9 (2018).

51. Lashley, T. et al. A pathogenic progranulin mutation and C9orf72 repeat expansion in a family with frontotemporal dementia. Neuropathol Appl Neurobiol 40, 502-13 (2014).

52. Mignarri, A. et al. Double trouble? Progranulin mutation and C9ORF72 repeat expansion in a case of primary non-fluent aphasia. J Neurol Sci 341, 176-8 (2014).

53. Testi, S., Tamburin, S., Zanette, G. \& Fabrizi, G.M. Co-occurrence of the C9ORF72 expansion and a novel GRN mutation in a family with alternative expression of frontotemporal dementia and amyotrophic lateral sclerosis. J Alzheimers Dis 44, 49-56 (2015).

54. Ciani, M. et al. The Missing Heritability of Sporadic Frontotemporal Dementia: New Insights from Rare Variants in Neurodegenerative Candidate Genes. Int J Mol Sci 20(2019).

55. Papadimitriou, S. et al. Predicting disease-causing variant combinations. Proc Natl Acad Sci U S A 116, 11878-11887 (2019).

56. Rossor, M.N., Fox, N.C., Mummery, C.J., Schott, J.M. \& Warren, J.D. The diagnosis of youngonset dementia. Lancet Neurol 9, 793-806 (2010).

57. Schindler, S.E. \& Fagan, A.M. Autosomal Dominant Alzheimer Disease: A Unique Resource to Study CSF Biomarker Changes in Preclinical AD. Front Neurol 6, 142 (2015).

58. Ryman, D.C. et al. Symptom onset in autosomal dominant Alzheimer disease: a systematic review and meta-analysis. Neurology 83, 253-60 (2014).

59. Fox, N.C. et al. Clinicopathological features of familial Alzheimer's disease associated with the M139V mutation in the presenilin 1 gene. Pedigree but not mutation specific age at onset provides evidence for a further genetic factor. Brain 120 ( Pt 3), 491-501 (1997).

60. Arboleda-Velasquez, J.F. et al. Resistance to autosomal dominant Alzheimer's disease in an APOE3 Christchurch homozygote: a case report. Nat Med 25, 1680-1683 (2019).

61. Thordardottir, S. et al. Reduced penetrance of the PSEN1 H163Y autosomal dominant Alzheimer mutation: a 22-year follow-up study. Alzheimers Res Ther 10, 45 (2018).

62. Rossor, M.N., Fox, N.C., Beck, J., Campbell, T.C. \& Collinge, J. Incomplete penetrance of familial Alzheimer's disease in a pedigree with a novel presenilin-1 gene mutation. Lancet 347, 1560 (1996).

63. Liang, Y. et al. A cognitive chameleon: lessons from a novel MAPT mutation case. Neurocase 20, 684-94 (2014).

64. Mead, S., Lloyd, S. \& Collinge, J. Genetic Factors in Mammalian Prion Diseases. Annu Rev Genet 53, 117-147 (2019).

65. Bernardi, L. et al. Novel PSEN1 and PGRN mutations in early-onset familial frontotemporal dementia. Neurobiol Aging 30, 1825-33 (2009).

66. Ramos, E.M. et al. Genetic screening of a large series of North American sporadic and familial frontotemporal dementia cases. Alzheimers Dement 16, 118-130 (2020).

67. Ryan, N.S. et al. Clinical phenotype and genetic associations in autosomal dominant familial Alzheimer's disease: a case series. Lancet Neurol 15, 1326-1335 (2016).

An excellent exploration of the genetic causes of early-onset Alzheimer's disease. 
68. Guven, G. et al. A patient with early-onset Alzheimer's disease with a novel PSEN1 p.Leu424Pro mutation. Neurobiol Aging 84, 238 e1-238 e4 (2019).

69. Carrasquillo, M.M. et al. Late-onset Alzheimer disease genetic variants in posterior cortical atrophy and posterior AD. Neurology 82, 1455-62 (2014).

70. Sitek, E.J. et al. A patient with posterior cortical atrophy possesses a novel mutation in the presenilin 1 gene. PLoS One 8, e61074 (2013).

71. Canevelli, M. et al. Familial Alzheimer's disease sustained by presenilin 2 mutations: systematic review of literature and genotype-phenotype correlation. Neurosci Biobehav Rev 42, 170-9 (2014).

72. Wallon, D. et al. The French series of autosomal dominant early onset Alzheimer's disease cases: mutation spectrum and cerebrospinal fluid biomarkers. J Alzheimers Dis 30, 847-56 (2012).

73. Ryan, N.S. et al. Genetic determinants of white matter hyperintensities and amyloid angiopathy in familial Alzheimer's disease. Neurobiol Aging 36, 3140-3151 (2015).

74. Shea, Y.F. et al. A systematic review of familial Alzheimer's disease: Differences in presentation of clinical features among three mutated genes and potential ethnic differences. J Formos Med Assoc 115, 67-75 (2016).

75. Voglein, J. et al. Clinical, pathophysiological and genetic features of motor symptoms in autosomal dominant Alzheimer's disease. Brain 142, 1429-1440 (2019).

76. Mann, D.M., Pickering-Brown, S.M., Takeuchi, A., Iwatsubo, T. \& Members of the Familial Alzheimer's Disease Pathology Study, G. Amyloid angiopathy and variability in amyloid beta deposition is determined by mutation position in presenilin-1-linked Alzheimer's disease. Am J Pathol 158, 2165-75 (2001).

77. McNaughton, D. et al. Duplication of amyloid precursor protein (APP), but not prion protein (PRNP) gene is a significant cause of early onset dementia in a large UK series. Neurobiol Aging 33, 426 e13-21 (2012).

78. Ryan, N.S. \& Rossor, M.N. Correlating familial Alzheimer's disease gene mutations with clinical phenotype. Biomark Med 4, 99-112 (2010).

79. Zarea, A. et al. Seizures in dominantly inherited Alzheimer disease. Neurology 87, 912-9 (2016).

80. Harold, D. et al. Genome-wide association study identifies variants at CLU and PICALM associated with Alzheimer's disease. Nat Genet 41, 1088-93 (2009).

81. Lambert, J.C. et al. Meta-analysis of 74,046 individuals identifies 11 new susceptibility loci for Alzheimer's disease. Nat Genet 45, 1452-8 (2013).

82. Guerreiro, R. et al. TREM2 variants in Alzheimer's disease. N Engl J Med 368, 117-27 (2013).

83. Hollingworth, P. et al. Common variants at ABCA7, MS4A6A/MS4A4E, EPHA1, CD33 and CD2AP are associated with Alzheimer's disease. Nat Genet 43, 429-35 (2011).

84. Escott-Price, V. et al. Common polygenic variation enhances risk prediction for Alzheimer's disease. Brain 138, 3673-84 (2015).

85. Wolf, A.B. et al. Apolipoprotein E as a beta-amyloid-independent factor in Alzheimer's disease. Alzheimers Res Ther 5, 38 (2013).

86. Belloy, M.E., Napolioni, V. \& Greicius, M.D. A Quarter Century of APOE and Alzheimer's Disease: Progress to Date and the Path Forward. Neuron 101, 820-838 (2019).

87. Uddin, M.S. et al. APOE and Alzheimer's Disease: Evidence Mounts that Targeting APOE4 may Combat Alzheimer's Pathogenesis. Mol Neurobiol 56, 2450-2465 (2019).

88. Bertram, L., McQueen, M.B., Mullin, K., Blacker, D. \& Tanzi, R.E. Systematic meta-analyses of Alzheimer disease genetic association studies: the AlzGene database. Nat Genet 39, 17-23 (2007).

89. Maestre, G. et al. Apolipoprotein E and Alzheimer's disease: ethnic variation in genotypic risks. Ann Neurol 37, 254-9 (1995). 
90. Kawamata, J., Tanaka, S., Shimohama, S., Ueda, K. \& Kimura, J. Apolipoprotein E polymorphism in Japanese patients with Alzheimer's disease or vascular dementia. J Neurol Neurosurg Psychiatry 57, 1414-6 (1994).

91. Reiman, E.M. et al. Exceptionally low likelihood of Alzheimer's dementia in APOE2 homozygotes from a 5,000-person neuropathological study. Nature Communications 11, 667 (2020).

In this study, neuropathological data was used to demonstrate the relative risk levels bestowed by the different ApoE genotypes.

92. Langlois, C.M. et al. Alzheimer's Prevention Initiative Generation Program: Development of an $A P O E$ genetic counseling and disclosure process in the context of clinical trials. Alzheimers Dement (N Y) 5, 705-716 (2019).

93. Coyle-Gilchrist, I.T. et al. Prevalence, characteristics, and survival of frontotemporal lobar degeneration syndromes. Neurology 86, 1736-43 (2016).

94. Rohrer, J.D. et al. The heritability and genetics of frontotemporal lobar degeneration. Neurology 73, 1451-6 (2009).

95. Mahoney, C.J. et al. Frontotemporal dementia with the C9ORF72 hexanucleotide repeat expansion: clinical, neuroanatomical and neuropathological features. Brain 135, 736-50 (2012).

96. Hutton, M. et al. Association of missense and 5'-splice-site mutations in tau with the inherited dementia FTDP-17. Nature 393, 702-5 (1998).

97. Cruts, M. et al. Null mutations in progranulin cause ubiquitin-positive frontotemporal dementia linked to chromosome 17q21. Nature 442, 920-4 (2006).

98. Greaves, C.V. \& Rohrer, J.D. An update on genetic frontotemporal dementia. J Neurol 266, 2075-2086 (2019).

99. Poos, J.M. et al. Cognitive profiles discriminate between genetic variants of behavioral frontotemporal dementia. J Neurol, 2020 Jun;267(6):1603-1612. doi: 10.1007/s00415-02009738-y. Epub 2020 Feb 12. (2020).

100. Moore, K.M. et al. Age at symptom onset and death and disease duration in genetic frontotemporal dementia: an international retrospective cohort study. Lancet Neurol, 2020 Feb;19(2):145-156. doi: 10.1016/S1474-4422(19)30394-1. Epub 2019 Dec 3. (2019).

An exploration of age at onset, disease duration and age at death in genetic frontotemporal dementia; a significant influence of family history was found.

101. Goldman, J.S. et al. Comparison of family histories in FTLD subtypes and related tauopathies. Neurology 65, 1817-9 (2005).

102. Po, K. et al. Heritability in frontotemporal dementia: more missing pieces? J Neurol 261, 2170-7 (2014).

103. Wood, E.M. et al. Development and validation of pedigree classification criteria for frontotemporal lobar degeneration. JAMA Neurol 70, 1411-7 (2013).

104. Rohrer, J.D. The genetics of primary progressive aphasia. Aphasiology 28, 941-947 (2014).

105. Turner, M.R. et al. Genetic screening in sporadic ALS and FTD. J Neurol Neurosurg Psychiatry 88, 1042-1044 (2017).

106. Rohrer, J.D. et al. Presymptomatic cognitive and neuroanatomical changes in genetic frontotemporal dementia in the Genetic Frontotemporal dementia Initiative (GENFI) study: a cross-sectional analysis. Lancet Neurol 14, 253-62 (2015).

107. Medicine, U.S.N.L.o. ARTFL LEFFTDS Vol. 2020 (ClinicalTrials.gov).

108. Medicine, U.S.N.L.o. A Study to Evaluate Safety of Long-term AL001 Dosing in FTD Patients. Vol. 2020.

109. Medicine, U.S.L.o. A First in Human Study in Healthy Volunteers and in Participants With Frontotemporal Dementia With Granulin Mutation. (ClinicalTrials.gov).

110. Medicine, U.S.L.o. Safety and Therapeutic Potential of the FDA-approved Drug Metformin for C9orf72 ALS/FTD. (ClinicalTrials.gov). 
111. Paucar, M. et al. Genotype-phenotype analysis in inherited prion disease with eight octapeptide repeat insertional mutation. Prion 7, 501-10 (2013).

112. Mead, S. \& Reilly, M.M. A new prion disease: relationship with central and peripheral amyloidoses. Nat Rev Neurol 11, 90-7 (2015).

113. Rawlins, M. Huntington's disease out of the closet? Lancet 376, 1372-3 (2010).

114. Bates, G.P. et al. Huntington disease. Nat Rev Dis Primers 1, 15005 (2015).

115. Ghosh, R. \& Tabrizi, S.J. Clinical Features of Huntington's Disease. Adv Exp Med Biol 1049, 128 (2018).

116. Ghosh, R. \& Tabrizi, S.J. Huntington disease. Handb Clin Neurol 147, 255-278 (2018).

117. Gusella, J.F. et al. A polymorphic DNA marker genetically linked to Huntington's disease. Nature 306, 234-8 (1983).

118. MacLeod, R. et al. Recommendations for the predictive genetic test in Huntington's disease. Clin Genet 83, 221-31 (2013).

The recommended protocol for how to proceed with counselling for predictive testing in genetic diseases.

119. A novel gene containing a trinucleotide repeat that is expanded and unstable on Huntington's disease chromosomes. The Huntington's Disease Collaborative Research Group. Cell 72, 971-83 (1993).

120. Rubinsztein, D.C. et al. Phenotypic characterization of individuals with 30-40 CAG repeats in the Huntington disease (HD) gene reveals HD cases with 36 repeats and apparently normal elderly individuals with 36-39 repeats. Am J Hum Genet 59, 16-22 (1996).

121. Bettencourt, C. et al. DNA repair pathways underlie a common genetic mechanism modulating onset in polyglutamine diseases. Ann Neurol 79, 983-90 (2016).

122. Becanovic, K. et al. A SNP in the HTT promoter alters NF-kappaB binding and is a bidirectional genetic modifier of Huntington disease. Nat Neurosci 18, 807-16 (2015).

123. Andrew, S.E. et al. Huntington disease without CAG expansion: phenocopies or errors in assignment? Am J Hum Genet 54, 852-63 (1994).

124. Wild, E.J. \& Tabrizi, S.J. Huntington's disease phenocopy syndromes. Curr Opin Neurol 20, 681-7 (2007).

125. Hensman Moss, D.J. et al. C9orf72 expansions are the most common genetic cause of Huntington disease phenocopies. Neurology 82, 292-9 (2014).

126. Richards, S. et al. Standards and guidelines for the interpretation of sequence variants: a joint consensus recommendation of the American College of Medical Genetics and Genomics and the Association for Molecular Pathology. Genet Med 17, 405-24 (2015).

The recommended guidelines on how to classify variants using all available evidence.

127. Vos, J. et al. The counsellees' view of an unclassified variant in BRCA1/2: recall, interpretation, and impact on life. Psychooncology 17, 822-30 (2008).

128. Ackerman, M.J. Genetic purgatory and the cardiac channelopathies: Exposing the variants of uncertain/unknown significance issue. Heart Rhythm 12, 2325-31 (2015).

129. Sobreira, N., Schiettecatte, F., Valle, D. \& Hamosh, A. GeneMatcher: a matching tool for connecting investigators with an interest in the same gene. Hum Mutat 36, 928-30 (2015).

130. Human Genetic Variation Database, http://www.hgvd.genome.med.kyoto-u.ac.jp/

131. Alzgene 09/2015, http://www.alzgene.org/

132. Guven, G. et al. Peripheral GRN mRNA and Serum Progranulin Levels as a Potential Indicator for Both the Presence of Splice Site Mutations and Individuals at Risk for Frontotemporal Dementia. J Alzheimers Dis 67, 159-167 (2019).

133. Cho Y, L.C., Jeong EG, et al. Prevalence of Rare Genetic Variations and their Implications in NGS-data Interpretation. Sci Rep 7(2017).

134. Yang, Y. et al. Molecular findings among patients referred for clinical whole-exome sequencing. JAMA 312, 1870-9 (2014). 
135. Yang, Y. et al. Clinical whole-exome sequencing for the diagnosis of mendelian disorders. $N$ Engl J Med 369, 1502-11 (2013).

136. Kalia, S.S. et al. Recommendations for reporting of secondary findings in clinical exome and genome sequencing, 2016 update (ACMG SF V2.0): a policy statement of the American College of Medical Genetics and Genomics. Genet Med 19, 249-255 (2017).

137. Amendola, L.M. et al. Performance of ACMG-AMP Variant-Interpretation Guidelines among Nine Laboratories in the Clinical Sequencing Exploratory Research Consortium. Am J Hum Genet 98, 1067-1076 (2016).

138. Dewey, F.E. et al. Distribution and clinical impact of functional variants in 50,726 wholeexome sequences from the DiscovEHR study. Science 354(2016).

139. Hart, M.R. et al. Secondary findings from clinical genomic sequencing: prevalence, patient perspectives, family history assessment, and health-care costs from a multisite study. Genet Med 21, 1100-1110 (2019).

140. Shahmirzadi, L. et al. Patient decisions for disclosure of secondary findings among the first 200 individuals undergoing clinical diagnostic exome sequencing. Genet Med 16, 395-9 (2014).

141. Hicks, J.K. et al. Patient Decisions to Receive Secondary Pharmacogenomic Findings and Development of a Multidisciplinary Practice Model to Integrate Results Into Patient Care. Clin Trans/ Sci 11, 71-76 (2018).

142. Daack-Hirsch, S. et al. 'Information is information': a public perspective on incidental findings in clinical and research genome-based testing. Clin Genet 84, 11-8 (2013).

143. Almqvist, E.W., Bloch, M., Brinkman, R., Craufurd, D. \& Hayden, M.R. A worldwide assessment of the frequency of suicide, suicide attempts, or psychiatric hospitalization after predictive testing for Huntington disease. Am J Hum Genet 64, 1293-304 (1999).

144. Owen, J. et al. Predictive testing for inherited prion disease: report of 22 years experience. Eur J Hum Genet 22, 1351-6 (2014).

145. ABC v St George's Hospital NHS Trust. in MRS JUSTICE YIP DBE http://www.bailii.org/ew/cases/EWHC/QB/2020/455.html (2020).

This judement clarified the duties of the treating clinicians when a patient refuses to tell their relatives of a pertinent genetic mutation; it has brought substantial change to clinical practice.

146. Florida. Supreme, C. Pate v. Threlkel. Wests South Report 661, 278-82 (1995).

147. Wiens, M.E., Wilson, B.J., Honeywell, C. \& Etchegary, H. A family genetic risk communication framework: guiding tool development in genetics health services. J Community Genet 4, 23342 (2013).

148. Consent and confidentiality in genomic medicine, 3rd Edition, (Joint Committee on Genomics in Medicine, https://www.bsgm.org.uk/joint-committee-on-genomics-in-medicine/, 2019).

149. Insurers, A.A.o.B. Genetics and insurance. (ABI Webpage).

150. Craufurd, D. \& Harper, P.S. Genetic counseling and genetic testing for neurogenetic disorders. Neurogenetics: A Guide for Clinicians, 6-16 (2012).

151. LaDuca, H. et al. Exome sequencing covers $>98 \%$ of mutations identified on targeted next generation sequencing panels. PLoS One 12, e0170843 (2017).

152. Pottier, C. et al. Genome-wide analyses as part of the international FTLD-TDP whole-genome sequencing consortium reveals novel disease risk factors and increases support for immune dysfunction in FTLD. Acta Neuropathol 137, 879-899 (2019).

153. Cacace, R., Sleegers, K. \& Van Broeckhoven, C. Molecular genetics of early-onset Alzheimer's disease revisited. Alzheimers Dement 12, 733-48 (2016).

This paper lays out the known mutations in APP, PSEN1 and PSEN2, and their location in relation to functional domains in great detail.

154. Rovelet-Lecrux, A. et al. APP locus duplication causes autosomal dominant early-onset Alzheimer disease with cerebral amyloid angiopathy. Nat Genet 38, 24-6 (2006). 
155. Neueder, A. et al. The pathogenic exon $1 \mathrm{HTT}$ protein is produced by incomplete splicing in Huntington's disease patients. Sci Rep 7, 1307 (2017).

156. Balendra, R. \& Isaacs, A.M. C9orf72-mediated ALS and FTD: multiple pathways to disease. Nat Rev Neurol 14, 544-558 (2018).

157. Santa-Maria, l. et al. The MAPT H1 haplotype is associated with tangle-predominant dementia. Acta Neuropathol 124, 693-704 (2012).

158. Forrest, S.L., Kril, J.J. \& Halliday, G.M. Cellular and regional vulnerability in frontotemporal tauopathies. Acta Neuropathol 138, 705-727 (2019).

\section{Acknowledgements}

C.K. is supported by a Leonard Wolfson Foundation PhD fellowship. N.S.R. is supported by a University of London Chadburn Academic Clinical Lectureship. J.D.R. is supported by a Medical Research Council Clinician Scientist Fellowship (MR/M008525/1) and has received funding from the NIHR Rare Disease Translational Research Collaboration (BRC149/NS/MH). J.M.S. acknowledges the support of the National Institute for Health Research University College London Hospitals Biomedical Research Centre, Wolfson Foundation, ARUK (ARUK-PG2017-1946), Brain Research UK (UCC14191, Weston Brain Institute (UB170045), Medical Research Council, British Heart Foundation and European Union's Horizon 2020 research and innovation programme (Grant 666992). N.C.F. acknowledges support from the UK Dementia Research Institute, from the Rosetrees Trust and from the NIHR Biomedical Research Centre at University College Hospitals NHS Foundation Trust. S.J.T. has received grant funding for her Huntington disease research from the Medical Research Council (UK), the Wellcome Trust, the Rosetrees Trust, Takeda Pharmaceuticals, Cantervale Limited, the NIHR North Thames Local Clinical Research Network, the UK Dementia Research Institute, the Wolfson Foundation for Neurodegeneration and the CHDI Foundation. S.M. is supported by the Medical Research Council (UK), the National Institute for Health Research Queen Square Dementia Biomedical Research Unit and the National Institute for Health Research Biomedical Research Centre at University College Hospitals NHS Foundation Trust.

\section{Author contributions}

All authors contributed to the drafting and proofreading of the manuscript. CK produced the figures and SM had the original idea for this review article.

\section{Competing interests}

The authors declare no competing interests.

\section{Peer review information}

Nature Reviews Neurology thanks J. Goldman and the other, anonymous, reviewer(s) for their contribution to the peer review of this work. 


\section{Key points}

- For typical dementia, appropriate genetic test for some groups is a gene panel and C9orf72 expansion testing, which balances the chance of discovery with costs and avoids variants of uncertain significance.

- Single-gene tests are only warranted in Huntington disease, prion disease or to confirm a known familial mutation; atypical syndromes necessitate whole-exome sequencing (WES) or whole-genome sequencing (WGS) and C9orf72 expansion testing.

- Discovery rates with WES and WGS are similar to those with gene panels but WES and WGS data can be re-analysed when new information becomes available.

- Uptake of predictive testing is currently low but will increase as treatment options become available because patients with a genetic diagnosis are ideal candidates for diseasemodifying drug trials.

- Additional tests are currently required to detect repeat expansions but long-read sequencing will enable simultaneous testing for single nucleotide polymorphisms and repeat expansions once sufficiently reliable and accurate.

- Genetic testing requires counselling on variants of uncertain significance, secondary findings and implications for relatives; refusal of a patient to share data with relatives could influence assessment of their decision-making capacity. 
Table 1 | Methods of gene testing

\begin{tabular}{|c|c|c|c|}
\hline Method & Technology & Advantages & Disadvantages \\
\hline \multicolumn{4}{|c|}{ Single Gene Testing } \\
\hline $\begin{array}{c}\text { Sanger } \\
\text { Sequencing }\end{array}$ & $\begin{array}{l}\text { PCR generates sequences of different } \\
\text { lengths with terminal } \\
\text { dideoxynucletotides, which are then } \\
\text { separated with capillary electrophoresis. }\end{array}$ & $\begin{array}{l}\text { High accuracy for SNPs, gold } \\
\text { standard for confirmatory testing }\end{array}$ & $\begin{array}{l}\text { Labour-intensive, slow, expensive } \\
\text { on a large scale, unsuitable for } \\
\text { detection of complex variants } \\
\text { such as copy number variants, } \\
\text { insertions and deletions }\end{array}$ \\
\hline $\begin{array}{l}\text { Fragment } \\
\text { analysis }\end{array}$ & $\begin{array}{l}\text { Repeat-primed PCR produces fragments } \\
\text { of the repeat, enabling the sizes of DNA } \\
\text { fragments from the region, including } \\
\text { pathogenic lengths, to be plotted }{ }^{25} \text {. }\end{array}$ & $\begin{array}{l}\text { Can detect nucleotide repeat } \\
\text { expansions even when extremely } \\
\text { large and intractable to simple } \\
\text { PCR-based approaches }\end{array}$ & $\begin{array}{l}\text { Labour intensive, not suitable for } \\
\text { detection of smaller variants }\end{array}$ \\
\hline Southern blot & $\begin{array}{l}\text { Oligonucleotide probes that match the } \\
\text { repeat expansion are hybridized to target } \\
\text { DNA fragments, washed, separated by } \\
\text { electrophoresis and visualized with a } \\
\text { luminescent dye }{ }^{26} \text {. }\end{array}$ & $\begin{array}{l}\text { Can detect and approximate the } \\
\text { size of nucleotide repeat } \\
\text { expansions, even when very large }\end{array}$ & $\begin{array}{l}\text { Labour intensive, not suitable for } \\
\text { detection of smaller variants }\end{array}$ \\
\hline \multicolumn{4}{|c|}{ Multiple Gene Testing } \\
\hline Method & Technology & Benefits and challenges & \\
\hline $\begin{array}{c}\text { Targeted } \\
\text { gene panels }\end{array}$ & $\begin{array}{l}\text { Amplicons of interest are selected using } \\
\text { primers and combined into a sample } \\
\text { library, which is amplified further, } \\
\text { sequenced and read on the sequencing } \\
\text { platform }{ }^{17} \text {; sequences are assembled by } \\
\text { aligning to a reference genome }\end{array}$ & $\begin{array}{l}\text { Targets genes relevant to a } \\
\text { phenotype, including rarer } \\
\text { causes; cheap and easy, high } \\
\text { accuracy for SNP detection and } \\
\text { small insertions and deletions, } \\
\text { can cover several related } \\
\text { conditions (for example, AD and } \\
\text { FTD) }\end{array}$ & $\begin{array}{l}\text { Mostly only suitable for } \\
\text { conditions for which most cases } \\
\text { are caused by a limited number } \\
\text { of genes; not suitable for repeat } \\
\text { disorders of copy number } \\
\text { variants, newly discovered genes } \\
\text { can only be incorporated after a } \\
\text { delay }\end{array}$ \\
\hline $\begin{array}{l}\text { Whole-exome } \\
\text { sequencing }\end{array}$ & $\begin{array}{l}\text { Similar to targeted gene panels but } \\
\text { selected amplicons cover all protein- } \\
\text { coding regions; samples are sequenced } \\
\text { on a high-throughput platform }{ }^{18} \text { and } \\
\text { sequences assembled to a reference } \\
\text { genome }\end{array}$ & $\begin{array}{l}\text { High benefit-cost ratio }{ }^{21,29} ; \\
\text { coverage approaches that of } \\
\text { gene panels }{ }^{151} \text {; high accuracy for } \\
\text { SNPs and small insertions and } \\
\text { deletions; useful in } \\
\text { heterogeneous disorders }{ }^{21} ; \text { no } \\
\text { bias; enables exon-based re- } \\
\text { analysis if new information } \\
\text { becomes available }\end{array}$ & $\begin{array}{l}\text { Risk of incidental findings in } \\
\text { unrelated genes } 33,34\end{array}$ \\
\hline $\begin{array}{l}\text { Whole- } \\
\text { genome } \\
\text { sequencing }\end{array}$ & $\begin{array}{l}\text { Usually amplicon-based but genome can } \\
\text { be sheared into fragments instead of PCR } \\
\text { step; sequenced on the same platforms } \\
\text { as whole-exome sequencing }{ }^{152} \text { and } \\
\text { sequences are assembled to a reference } \\
\text { genome }\end{array}$ & $\begin{array}{l}\text { High accuracy for SNPs and small } \\
\text { insertions and deletions; } \\
\text { particularly useful for } \\
\text { hetereogeneous disorders; no } \\
\text { bias; enables re-analysis if new } \\
\text { information becomes available; } \\
\text { PCR-free sequencing enables } \\
\text { detection of copy number } \\
\text { variants }^{32}\end{array}$ & $\begin{array}{l}\text { High cost, especially with higher } \\
\text { coverage; risk of incidental } \\
\text { findings in unrelated genes }\end{array}$ \\
\hline $\begin{array}{l}\text { Long-read } \\
\text { sequencing }\end{array}$ & $\begin{array}{l}\text { Zero-mode waveguide used to create a } \\
\text { single-nucleotide observation space with } \\
\text { a DNA template and a polymerase; each } \\
\text { time a nucleotide is incorporated, the } \\
\text { zero-mode waveguide detects the } \\
\text { change in fluorescence related to the loss } \\
\text { of fluorescent dye. }\end{array}$ & $\begin{array}{l}\text { Can achieve read lengths of } \\
\text { several thousand base pairs so } \\
\text { can detect complex variants and } \\
\text { tandem repeats }{ }^{33} \text {; the best } \\
\text { method to date for sequencing } \\
\text { through repeat expansions }\end{array}$ & $\begin{array}{l}\text { Not as accurate as existing } \\
\text { platforms for detection of SNPs; } \\
\text { expensive }\end{array}$ \\
\hline $\begin{array}{l}\text { Oxford } \\
\text { Nanopore } \\
\text { Technologies } \\
\text { (ONT) } \\
\text { sequencing }\end{array}$ & $\begin{array}{l}\text { Protein nanopores are set in an } \\
\text { electrically resistant membrane, through } \\
\text { which an ionic current is passed, creating } \\
\text { a constant voltage; as the DNA strand } \\
\text { passes through the pore, each nucleotide } \\
\text { creates a distinct change in current. }\end{array}$ & $\begin{array}{l}\text { Can achieve read lengths of } \\
\text { several thousand base pairs so } \\
\text { can detect complex variants and } \\
\text { tandem repeats }{ }^{33} \text {; sequencing } \\
\text { device is small, cheap and } \\
\text { portable and can be scaled up }\end{array}$ & $\begin{array}{l}\text { Not as accurate as existing } \\
\text { platforms for detection of SNPs }\end{array}$ \\
\hline
\end{tabular}


AD, Alzheimer disease; FTD, frontotemporal dementia; PCR, polymerase chain reaction; SNP, single nucleotide polymorphism. 
Table 1 | Mutation types found in different forms of dementia

\begin{tabular}{|l|l|l|}
\hline Mutation type & Examples & Possible mechanism of effect \\
\hline Single nucleotide variation & $\begin{array}{l}\text { Missense mutations in APP, } \\
\text { PSEN1 and PSEN2 cause AD }\end{array}$ & $\begin{array}{l}\text { Increased production or } \\
\text { amyloidogenicity of amyloid- } \beta \\
\text { peptides }\end{array}$ \\
\hline Small insertions and deletions & $\begin{array}{l}\text { Insertions and deletions in } \\
\text { PSEN1 cause AD }\end{array}$ & $\begin{array}{l}\text { Increased production or } \\
\text { amyloidogenicity of amyloid- } \beta \\
\text { peptides }\end{array}$ \\
\hline $\begin{array}{l}\text { Large exon deletions, insertions, } \\
\text { duplications and copy number } \\
\text { variants }\end{array}$ & $\begin{array}{l}\text { APP duplication (including } \\
\text { trisomy 21) causes causes AD }\end{array}$ & $\begin{array}{l}\text { Increased expression of } \\
\text { amyloid- } \beta \text { peptides }\end{array}$ \\
\hline Splice-site mutations & $\begin{array}{l}\text { Splice-site mutations in GRN } \\
\text { and MAPT cause FTD }\end{array}$ & $\begin{array}{l}\text { Loss of GRN function } \\
\text { differential splicing of } \text { MAPT } \\
\text { causes an imbalance between } \\
\text { 3-repeat and 4-repeat tau }\end{array}$ \\
\hline Repeat expansions & $\begin{array}{l}\text { CAG expansion in HTT } \\
\text { causesHD, and GGGGCC } \\
\text { repeat expansion in C9orf72 } \\
\text { causes FTD, and ALS and HD- } \\
\text { like syndromes }\end{array}$ & $\begin{array}{l}\text { Gain-of-function and } \\
\text { differential splicing of } \\
H^{114,155} \text {; gain-of-function or } \\
\text { possibly loss-of-function of } \\
\text { C9orf72 }^{156}\end{array}$ \\
\hline Chromosomal region inversions & $\begin{array}{l}\text { Genomic inversion of MAPT } \\
\text { is a risk factor (with low } \\
\text { penetrance) for tangle- } \\
\text { dominant dementia }\end{array}$ & $\begin{array}{l}\text { Causes formation of } \\
\text { extracellular 3-repeat and 4- } \\
\text { repeat tau tangles }\end{array}$ \\
\hline
\end{tabular}

ALS, amyotrophic lateral sclerosis; AD, Alzheimer disease; FTD, frontotemporal dementia; HD, Huntington disease. 
Figure 1 | Diagnostic uncertainty and pleiotropy in dementia. Among patients with each clinical presentation of dementia on the left, a proportion have deleterious variants in genes normally associated with other diseases, shown on the right the lines indicate the crossover and their thicknesses are proportional to the proportion of patients in each group that carry deleterious variants in each gene category. Alzheimer disease can often be used as a default or provisional diagnosis for patients with dementia, so a large proportion of patients with a clinical diagnosis of $A D$ have gene variants that are typically associated with other disorders. Frontotemporal dementia encompasses diverse clinical presentations and can consequently be mistaken for other dementia disorders. Dementia with motor symptoms, which encompasses atypical dementia syndromes with additional hypokinetic or hyperkinetic motor symptoms that are not caused by Huntington disease, can be difficult to diagnose and classify so have diverse genetic causes. For prion diseases, diagnostic rates for single-gene tests are consistently high. Atypical genes are genes that have been linked to dementias but are uncommon causes or cause additional features. Modified with permission from ref. ${ }^{24}$.

Figure 2 | Algorithm for genetic testing of patients with dementia. The likelihood that patients with dementia have a genetic cause for their disease can be stratified on the basis of their clinical phenotype, their age at onset and their family history expressed as the modified Goldman score (Box 2). We recommend testing all patients with Alzheimer disease (AD) and a strong family history or early onset disease ( $<60$ years; with the exception of posterior cortical atrophy (PCA), which is not usually associated with one of the typical $A D$ genes), all patients with behavioural variant frontotemporal dementia FTD (bvFTD) or FTD-amyotrophic lateral sclerosis (ALS), and patients with primary progressive aphasia (PPA) or a corticobasal syndrome (CBS) and a positive family history. Patients with Huntington disease (HD) or prion disease phenotypes should undergo a single gene test for the relevant condition first and if this test is negative and their modified Goldman score is $<3$, whole-exome sequencing (WES) or whole-genome sequencing (WGS) should be performed. Patients who do not meet any of the above criteria but have early-onset dementia or a relative with early-onset dementia should be considered for genetic testing, subject to other factors, such as limited information about the family history or suggestive disorders in the family. Patients with AD, age of onset $>65$ years and no family history should generally not be offered genetic testing. Modified with permission from ref $^{24}$.

Box 1 | Limits of short-read sequencing

Current clinically used next-generation sequencing platforms depend on DNA reading by sequencing and automated detection of light or radioactivity as nucleotides are incorporated. Typically, sequenced fragments reach several hundred base pairs before the DNA polymerase detaches. The complete sequence is then assembled from these fragments by matching regions that overlap. Tandem repeats are, by nature, highly repetitive, and repeat expansions consequently render the fragment assembly virtually impossible because the position of each fragment cannot be determined. This limitation prevents detection of repeat expansions, especially if they are novel; it also limits the identification of breakpoints for structural variants if they fall into a repetitive region. Paired-read sequencing compensates for these limitations to some extent but is not reliable enough for clinical use.

Box 2 | The modified Goldman score

The modified Goldman score enables stratification of a family history based on the number of a patient's relatives who are or were affected. The score strongly correlates with the likelihood of identifying a causal mutation ${ }^{94}$. A modified Goldman score of 1 corresponds to a family history of symptoms consistent with the clinical syndrome with an autosomal dominant inheritance pattern, with at least three people who are affected in two generations and who are linked by a first-degree relative. A modified Goldman score of 2 indicates familial aggregation of three of more affected relatives but without meeting the criteria for a score of. A modified Goldman score of 3 denotes one other affected relative (the score is 3 if the age of onset is $<65$ years or 3.5 if the age at onset is $>65$ years). A modified Goldman score of 4 signifies no known family history of neurodegenerative disease ${ }^{94}$.

Box 3 | Application of the ACMG-AMP guidelines

The American College of Medical Genetics and Association of Molecular Pathology (ACMG-AMP) guidelines for classification of variants are designed to combine several pieces of evidence for accurate classification. Each piece of evidence is itself classified according to its level of reliability and whether it indicates pathogenicity. 
The classification of each variant then depends on the sum of all available evidence. For example, a variant can only be classified as pathogenic if at least two strong pieces of evidence indicate its pathogenicity. If these criteria are not met, or if evidence is contradictory, the variant remains a variant of uncertain significance. The complexity means that multi-disciplinary teams, including clinicians and geneticists, are often required to classify variants. Examples of evidence that is used to classify variants are given below:

\section{Evidence that a variant is pathogenic}

- The variant is a coding amino acid change that has previously been identified as deleterious.

- The variant is absent or very infrequent in population databases.

- The variant is in a mutational hotspot.

- Two or more independent in silico prediction models suggest that the variant is damaging.

\section{Evidence that a variant is benign}

- The variant is common in population databases.

- Previous studies have indicated that the variant is benign

- The allele frequency is greater than expected in the general population to be a fully penetrant cause of a rare disorder.

\section{Box 4 | Benefits and detrimental effects of a genetic diagnosis}

\section{Beneficial effects}

- Provides diagnostic near certainty and enables adequate disease monitoring

- Further diagnostic tests are unnecessary

- Enables initiation of symptomatic treatment and discontinuation of ineffective treatments

- Facilitates reproductive strategies, such as in vitro fertilization with pre-implantation genetic diagnosis or invasive genetic testing in pregnancy

- Provides access to support groups, clinical trials and targeted treatments when they become available

\section{Detrimental effects}

- Can cause psychosocial difficulties

- Can lead to the breakdown of social relationships

- Identification of pathogenic mutations can affect job and insurance prospects

- Identification of a variant of uncertain significance neither excludes genetic disease nor permits predictive testing for relatives ${ }^{48,150}$

\section{Glossary terms}

\section{Allelic drop out}

Failure to amplify one or both alleles during a sequencing reaction.

\section{Breakpoints}

Limits or borders of a structural variant where they link to the surrounding normal genomic sequence.

\section{Paired-read sequencing}

The process of sequencing a genomic fragment using adapters to both ends of the fragment, which improves reference sequence alignment and facilitates the analysis of repetitive regions. 


\section{Segregation analysis}

Genetic analysis of affected and unaffected members of a family for their carrier status with regards to a particular genetic variant.

\section{Anticipation}

A phenomenon in which age at onset decreases and severity of phenotype increases from one generation to the next in some genetic diseases; typical of some trinucleotide repeat disorders in which the number of repeats is linked to the age at onset and the severity of disease.

In this Review, the authors discuss how technological advances are enabling clinical genetic testing for various dementia disorders, consider which types of tests are appropriate for which patients, and look at the ethical issues that can be raised by genetic testing in these disorders. 